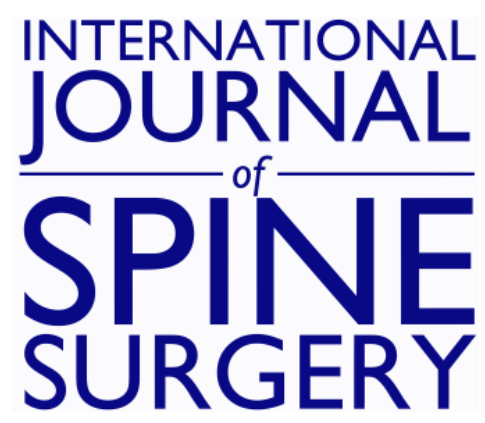

\title{
Readmissions After Outpatient Transforaminal Decompression for Lumbar Foraminal and Lateral Recess Stenosis
}

\section{KAI-UWE LEWANDROWSKI}

Int J Spine Surg 2018, 12 (3) 342-351

doi: https://doi.org/10.14444/5040

http://ijssurgery.com/content/12/3/342

This information is current as of April 26, 2023.

Email Alerts Receive free email-alerts when new articles cite this article. Sign up at: http://ijssurgery.com/alerts 


\title{
Readmissions After Outpatient Transforaminal Decompression for Lumbar Foraminal and Lateral Recess Stenosis
}

\author{
KAI-UWE LEWANDROWSKI, MD \\ Center for Advanced Spine Care of Southern Arizona, Tucson, Arizona; University of Arizona, Tucson, Arizona; Department of Neurosurgery, Universidade \\ Federal do Estado do Rio de Janeiro-UNIRIO, Rio de Janeiro, Brazil
}

\begin{abstract}
Background: The objective of this study was to analyze readmission rates after outpatient transforaminal endoscopic decompression surgery for lumbar foraminal and lateral recess stenosis done in an ambulatory surgery center. Endoscopic lumbar spinal surgery is gaining popularity for the treatment of lumbar disc herniations. Recent advances in surgical techniques allow for percutaneous endoscopically assisted bony decompression for neurogenic claudication symptoms due to spinal stenosis. The surgery can be done under local anesthesia and sedation. Patients may be discharged home within hours from surgery, and complications are rare. However, readmissions for recurrent disc herniations, failure of pain relief, dysesthetic leg pain, nerve root injuries with foot drop, and facet and pedicle fractures have been reported.

Methods: A retrospective study of 1839 consecutive patients with an average mean follow up of 33 months (range 24 to 85 months) that underwent percutaneous endoscopic surgery at 2076 levels between 2006 and 2015 was conducted with the intent of identifying factors associated with emergency room or hospital readmission following endoscopic foraminotomy and microdiscectomy. Only patients with unilateral radiculopathy due to either herniated disc or lateral recess stenosis were included in this study. Preoperatively, disc migration was graded by direction and distance from the disc space according to Lee's radiologic 4-zone classification. The type of disc herniation was classified either as extruded or contained. Contained herniations were further subclassified as disc protrusions versus disc bulges. In addition, the preoperative disc height was recorded. Bony spinal foraminal stenosis and lateral recess stenosis were graded on preoperative magnetic resonance imaging and computed tomography scans into mild, moderate, and severe by dividing the lumbar neuroforamen into 3 zones: (a) entry zone, (b) midzone, and (c) exit zone. Surgical outcomes were classified according to the Macnab criteria. In addition, reduction in visual analog scores (VASs) were assessed. The treating physician (KUL) performed all surgeries.

Results: According to the Macnab criteria, excellent and good results were obtained in $82.2 \%$ of patients with extruded disc fragment (331/1839). In this group, the mean VAS decreased from $5.9 \pm 2.5$ preoperatively to $2.4 \pm 1.8$ at the final follow up $(P<.01)$. Patients with contained disc herniations $(648 / 1839)$ had excellent and good results $72.7 \%$ of the time. In this group, the mean VAS decreased from $7.2 \pm 1.6$ preoperatively to $3.1 \pm 1.5$ at the final follow up $(P<$ $.01)$. In the spinal stenosis group (860/1839), $75 \%$ of patients had excellent to good results. There were no major approach-related complications. Sixty-nine patients had extravasations of irrigation fluid into the subcutaneous tissues $(3.8 \%)$. Eight patients developed spinal headaches $(0.4 \%)$. Two patients developed foot drop on the surgical side immediately postoperatively $(0.1 \%)$. Reherniations of extruded discs occurred in 9 patients $(2.7 \%$ recurrence rate). Failure of pain relief without significant improvement of walking endurance occurred in 29 patients with bony stenosis in the central canal, lateral recess, and entry zone of the neuroforamen $(3.3 \%)$. Reherniations were associated with preserved disc height of $>6 \mathrm{~mm}(P<.02)$. Dysesthetic leg pain due to dorsal root ganglion irritation occurred in 229 patients $(12.4 \%)$ and was unrelated to case frequency but was associated with severe foraminal stenosis $(P<.01)$. All 229 patients improved with supportive care. Facet or pedicle fractures did not occur in this series. There were 26 acute care (within 6 weeks from surgery) postoperative emergency room visits [16 of which resulted in readmission to a hospital over the 9year study period $(0.86 \%)$ : 9 for dysesthetic leg pain, 2 for wound infections, and 5 for poorly controlled incisional pain].

Conclusions: Transforaminal endoscopic decompression can be successfully carried out in an outpatient surgery center setting. Readmissions due to reherniations, postoperative complications, or poor pain control are uncommon.
\end{abstract}

Endoscopic Minimally Invasive Surgery

Keywords: lumbar endoscopic decompression, complications, readmissions 


\section{INTRODUCTION}

Microdiscectomy is a well-established surgical decompression procedure for symptomatic herniations of the lumbar intervertebral disc refractory to conservative care measures including physical therapy, nonsteroidal anti-inflammatory drugs, activity modification, and interventional spinal injection treatments. $^{1-5}$ Minimally invasive techniques have become mainstream in spinal surgery due to technological advances, such as retractors designed for intermuscular plane mini-open incisional exposures $^{6-8}$ or tubular access retractors, which in the case of lumbar spinal endoscopy have been further miniaturized into working cannulas just large enough to accommodate an endoscope. ${ }^{9-13}$ Less approach-related access trauma and reduced surgical pain in combination with an overall push by patients, insurance providers, and governmental review boards to transition simple lumbar decompression surgeries into a more cost-effective outpatient setting have facilitated a substantial increase of these types of procedures being carried out in an ambulatory surgery center (ASC). ${ }^{14,15}$

The value proposition is obvious: Lumbar decompression procedures can be performed at a lower cost in a lower-risk ASC setting with improved clinical outcomes; patients experience fewer postoperative complications, shorter intervals to return to work, social reintegration, shorter postoperative narcotic independence, and an overall reduced utilization of pain killers. The latter problem is of significance in the context of the narcotic abuse epidemic in the United States and has prompted increasing scrutiny by patients, insurance providers, and governmental review boards to employ a more stringent medical necessity and cost-benefit assessment system to both inpatient and outpatient spinal surgeries. ${ }^{16-19}$ Utilization of evidence-based treatments is demanded by many to keep the rising cost of lumbar spinal surgery in check at a time where there is a growing demand for these types of procedures because of an aging baby boomer population. ${ }^{20-22}$

Recently, incentives to provide more cost-effective, high-value spinal care have been put in place in the form of new current procedural terminology (CPT) codes making a large number of spinal surgeries contractually feasible in an outpatient ASC. $^{23}$ Bundled payments for care improvement (BPCI) models have been implemented in total joint arthroplasty to reduce costs by allotting a fixed payment for an episode of care to incentivize hospitals to implement core measures to decrease length of stay, the incidence of wound infections, improve integrated management of medical comorbidities, and reduce visits to the emergency room or readmission to the hospital, as well as reducing the number of patients sent to inpatient rehabilitation facilities for continued postoperative care. ${ }^{24,25}$ Implementation of similar strategies may be on the horizon for the increasing number of ASC spinal surgery procedures that makes understanding factors contributing to readmissions crucial in the overall design of a value-based outpatient spinal care program. ${ }^{26}$

The aim of this study was to simply investigate whether the outpatient lumbar transforaminal decompression procedure when done in an ASC for herniated disc and spinal stenosis can adequately address sciatica-type leg and low back pain symptoms without substantial need for additional inpatient aftercare or readmission to an emergency room or hospital because of reherniations, postoperative complications, or poor pain control.

\section{MATERIALS AND METHODS}

In 2006, the Center for Advanced Spine Care of Southern Arizona established an outpatient spinal surgery program for the treatment of lumbar herniated disc and spinal stenosis. The results presented in this paper are based on a retrospective review of patients that were seen by the treating surgeon (KUL) between the years 2006 and 2015. Patients were worked up diagnostically by reviewing prior referrals to consulting physicians, pain management, and interventional care to integrate the information into clinical pathways to determine what additional diagnostic study or nonoperative treatment measure may be appropriate prior to considering surgical treatment. Patients were seen for lumbar radiculopathy with and without claudication and low back pain.

\section{Patient Population}

All patients in this case series provided informed consent. This retrospective study included a group of 1839 consecutive patients seen in our clinic who underwent percutaneous endoscopic foraminotomy and microdiscectomy at 2076 levels between 2006 and 2015. The mean follow up was 33 months ranging from 24 to 85 months at the time this study 
was concluded. The inclusion criteria were: (1) clinical signs of unilateral lumbar radiculopathy, dysesthesias, and decreased motor function; (2) imaging evidence of foraminal or lateral recess stenosis (criteria described below) demonstrated on preoperative magnetic resonance images (MRI) and computed tomography (CT) scans; (3) unsuccessful nonoperative treatment including physical therapy and transforaminal epidural steroid injections for at least 12 weeks; and (4) an age of 30-85 years. Patients exhibiting pain syndromes involving more than 1 dermatome or had bilateral symptoms, showed segmental instability on preoperative extension flexion radiographs, or had severe central stenosis (less than $100 \mathrm{~mm}^{2}$ ) or both were excluded from this study. ${ }^{27}$ Inclusion/exclusion criteria were used with the intent of minimizing the effect of other confounding factors. Patients' average age was 50.7 \pm 18.8 years. Of the 1839 patients selected, 1072 were female, and 767 were male.

\section{Preoperative Work Up and Clinical Follow Up}

Radiographs, MRI, and CT images were obtained preoperatively for all surgical patients. Typically, patients returned for clinical follow up at 6 weeks postoperatively, and at 3, 6, 12, and 24 months, respectively. After the 2-year follow-up appointment, patients were seen on an annual or biannual basis. The long-term follow up after 2 years was less reliable and available in only $81 \%$ of patients at 3 years and $68 \%$ at 4 years postoperatively. Therefore, results reported herein were computed from data obtained at 2-year follow up. Primary clinical outcome measures were reductions in the visual analog score (VAS) for leg pain ranging from no pain (0) to worst pain (10) and the Oswestry Disability Index (ODI) both done by the patient and by the treating surgeon (KUL) using the Macnab criteria. ${ }^{28}$ Briefly, follow-up results were classified as excellent if the patient had little pain and returned to desired activities with few limitations. Outcomes were classified as good if the patient reported occasional pain or dysesthesias with daily activities with minor restrictions and did not need any pain medication. Patients were assigned to 1 of the 2 remaining categories if their pain improved somewhat but they continued to need pain medication (fair) or if their function worsened or they needed additional surgery to address their symptoms (poor).

\section{Radiologic Classification of Foraminal Stenosis}

Lee's classification of foraminal and lateral recess stenosis was used to define the location of the offending pathology within the neuroforamen by dividing it from medial to lateral into entry (dura to pedicle; zone 1), middle (medial pedicle wall to center pedicle; zone 2), and exit zone (center pedicle to lateral border of the facet joint; zone 3). ${ }^{29}$ Foraminal and lateral recess stenosis were stratified according to the main offending pathology: extruded herniated disc, disc bulge, and disc bulge with concomitant bony stenosis. Disc herniations were further classified as upward, downward, migrated, or centered around this disc space using Lee's 4zone classification. ${ }^{30}$ In the entry zone, Lee described hypertrophy of the superior articular facet as the predominant pathology. ${ }^{29}$ In the midzone, it was often due to an osteophytic process underneath the pars interarticularis, and in the exit zone, due to a subluxed and hypertrophic facet joint. ${ }^{29}$ These classification systems have been previously applied by the author. ${ }^{9}$ The height of the posterior intervertebral disc and lumbar foramina was evaluated according to Hasegawa, ${ }^{31}$ who described a lumbar neuroforaminal height of $15 \mathrm{~mm}$ or more as normal and reduced posterior intervertebral disc height of 3 to $4 \mathrm{~mm}$ as suggestive of spinal stenosis. Preoperative sagittal and axial MRI and CT images were used to assess the location and extent of foraminal stenosis. Only patients with stenotic lesions (whether due to bony stenosis or extruded disc herniation or contained disc bulge) producing a neuroforaminal width of $3 \mathrm{~mm}$ or less on the sagittal MRI and CT cuts or lateral recess height of $3 \mathrm{~mm}$ or less on the axial MRI and CT cuts were treated surgically. The degree of lumbar intervertebral disc degeneration was also graded using the MRI classification system published by Pfirrmann et $\mathrm{al}^{32}$ with Grade I characterizing the MRI appearance of the surgical disc as ". . . homogeneous with bright hyperintense white signal intensity and normal disc height. ..", and Grade V as an inhomogeneous disc ". . . with hypointense black signal intensity. There is no difference between the nucleus and annulus. The disc space is collapsed. .."32

\section{Surgical Techniques}

All surgical procedures employed the endoscopic transforaminal approach using the "outside-in" technique, in which the working sheath is placed 
into the lower portion of the neuroforamen, thus retracting and avoiding the exiting nerve root. No part of the cannula tip or the endoscope is positioned in the disc space. The surgical technique used by the author (originally popularized by Hoogland and Schubert et al) ${ }^{33,34}$ employs a foraminoplasty in patients with or without lateral stenosis for the treatment of herniated disc.

Procedures were performed in prone position under local anesthesia and sedation in all patients. In some instances, where access to the L5/S1 neuroforamen was difficult due to a high riding ilium, patients were positioned in the lateral decubitus position. Techniques to define the skin entry point and the surgical trajectory have been described in previous literature. ${ }^{35-38}$ Generally, entry points were laterally at $7-9 \mathrm{~cm}$ at the $\mathrm{L} 3 / 4$ level, $8-10 \mathrm{~cm}$ at the $\mathrm{L} 4 / 5$ level, and $10-12 \mathrm{~cm}$ at the L5/S1 level.

The targeted neuroforamen was accessed as follows: First, an 18-G (150 mm in length) needle is inserted into the safe zone of Kambin's triangle bordered by the traversing nerve root medially, the exiting nerve root laterally, and the lower adjacent pedicle distally. ${ }^{35,36}$ Ideally, the targeting needle was placed on the lateral view into the lower portion of the neuroforamen or into the disc. On the anteriorposterior view, the needle tip should be at the medial interpedicular line. A steel guide wire was then inserted, and the 18-G spinal needle was removed. Dilators, drills, and trephines of increasing diameters were used for foraminal decompression procedures. Additional cannulated reamers measuring 7 and $9 \mathrm{~mm}$ in diameter intended to be used over a guide wire without the protective working cannula were available but rarely used to further minimize risk of dysesthesia of the exiting nerve root and irritation of its dorsal root ganglion (DRG).

For the foraminoplasty, bone from the hypertrophied superior and inferior articular process was removed with different instruments including endoscopic chisels, drills, Kerrison rongeurs, and percutaneous trephines. The endoscopic drills and rongeurs were deployed inside the center working cannula of the endoscope to lessen the risk of dysesthesia and irritation of the exiting nerve root and its DRG. In other words, the entire decompression was performed under continuous direct video-endoscopic visualization, and no part of the decompression procedure is done percutaneously with only indirect fluoroscopic imaging.
The foraminoplasty was facilitated by changing the trajectory of the instruments to aim for the compressive pathology identified on preoperative studies. In case of concomitant herniated disc, extruded disc material was removed using forceps and pituitary rongeurs, and contained herniations were decompressed through a small annular window. Epidural bleeding was controlled with a radiofrequency probe (Ellman, Ellman International, Hicksville, New York) under saline irrigation.

\section{Postoperative Rehabilitation and Utilization}

Postoperative rehabilitation and supportive care requirements were recorded and analyzed in relation to clinical outcomes with the secondary transforaminal endoscopic decompression procedure in lumbar monoradiculopathy patients due to lateral stenosis with or without herniated disc. During their regular postoperative visits, patients were asked whether they participated in any active exercise programs, physical or occupational therapy, chiropractic care, or had acupuncture or spinal injection treatments. In addition, the patients' utilization of nonsteroidal anti-inflammatories, narcotic, and other types of pain medication was recorded. Finally, patients were asked whether they developed any new pain syndromes or hitherto unknown conditions that negatively impacted their walking endurance.

\section{Readmission Analysis}

At each follow-up visit, patients were asked whether they were seen in an emergency room or admitted to a hospital following their transforaminal decompression procedure. Visits to an emergency room with or without admission to a hospital in the immediate postoperative period during the first 6 weeks after the index surgery were of particular interest. These visits were considered acute care visits and were a result of poor postoperative pain control, a postoperative complication such as new onset of neurological deficit, or a wound infection with fever and drainage from the wound. Emergency room visits or hospital admissions after 6 weeks from the transforaminal index decompression procedure were considered nonacute, chronic care visits. The reason for readmission and postoperative complications were recorded and analyzed in relation to the patient's overall functional outcome. 
Table 1. Patients by diagnosis $(\mathrm{N}=1839)$.

\begin{tabular}{lc}
\hline Indication for Surgery & Number of Patients \\
\hline Lumbar disc herniations & \\
Extruded & 331 \\
Contained & 648 \\
Subtotal & 979 \\
Lateral recess stenosis & 860 \\
Total & 1839 \\
\hline
\end{tabular}

\section{Statistical Methods}

For the clinical outcomes analysis, cross-tabulation statistics and measures of association were computed for 2-way tables using IBM SPSS Statistics software, Version 15.0. Using patient satisfaction data and clinical outcomes data based on the modified Macnab criteria, VAS, and foraminal zone classification, MRI classification of disc degeneration, definition of the location of any herniated disc, and foraminal height and width parameters as row and column variables, and age (over 50 and under 50 years of age) as control variable (layer factor), the cross-tabulation procedure was employed to form 1 panel of associated statistics and measures for each value of the layer factor (or a combination of values for 2 or more control variables). This correlation matrix allowed calculation of variable combinations if no association was found between readmission, clinical outcome and variable distribution was equal. Both the Pearson $\chi^{2}$ and the likelihood-ratio $\chi^{2}$ tests were used as statistical measures of association.

\section{RESULTS}

Of the 1839 patients that underwent outpatient decompression between 2006 and 2015 (Table 1), excellent and good results according to the Macnab criteria were obtained in $82.2 \%$ of patients with extruded disc fragment (331/1839). In this group, the mean VAS decreased from $5.9 \pm 2.5$ preoperatively to $2.4 \pm 1.8$ at the final follow up $(P<.01)$. Patients with contained disc herniations $(648 / 1839)$ had excellent and good results $69.7 \%$ of the time. In this group, the mean VAS decreased from $7.2 \pm 1.6$ preoperatively to $3.1 \pm 1.5$ at final follow up $(P<$ $.01)$. In the spinal stenosis group $(860 / 1839), 75 \%$ of patients had excellent to good results. There were no major approach- or anesthesia-related complications. Incidental durotomies were encountered in 2 patients, both in the axilla between the exiting L4 and the traversing L5 nerve. One of these 2 patients had a rootlet herniation that was reduced intraop- eratively. Both patients were successfully treated with a blood patch and bed rest for 24 hours. Neither of the 2 complained of spinal headaches. Sixty-nine patients had extravasations of irrigation fluid into the subcutaneous tissues $(3.8 \%)$. Eight patients developed spinal headaches $(0.4 \%)$. Two patients treated for lateral recess stenosis developed foot drop on the surgical side immediately postoperatively $(0.1 \%)$, both after a $\mathrm{L} 4 / 5$ decompression surgery. These 2 patients were reassured and sent home from the ASC with a prescription for an ankle-foot orthosis (AFO). One patient had noninsulin-dependent diabetes mellitus (NIDDM), and the other one did not. The NIDDM patient improved somewhat from a $2 / 5$ motor strength examination for extensor hallucis longus (EHL) and tibialis anterior (TA) muscles immediately postoperatively to a $4 / 5$ motor strength at 9 months followup visit. This patient was initially managed with an AFO and physical therapy focusing on strengthening both the EHL and TA muscles and on improving proprioception in the affected extremity. The other patient with EHL and TA weakness (4/5) had transitory motor dysfunction that improved spontaneously within 6 weeks from the surgery with physical therapy and self-directed supportive care measures without ultimately needing an orthosis.

Reherniations occurred in 9 of 331 patients with extruded disc herniations (2.7\% recurrence rate). Reherniations were associated with preserved disc height of $>6 \mathrm{~mm}(P<.02)$. Failure of pain relief without significant improvement of walking endurance occurred in 39 of the 860 patients with bony stenosis in the central canal, lateral recess, and entry zone of the neuroforamen $(4.5 \%)$ and in 41 of the 648 patients with contained disc bulges $(6.3 \%)$. The latter subset of patients with contained disc herniation had advanced degenerative changes. According to the MRI grading system published by Pfirrmann et al, nearly all the failed patients with contained disc herniations received Grade $\mathrm{V}$ and some Grade IV to characterize the advanced degeneration of the surgical lumbar disc. Dysesthetic leg pain due to DRG irritation occurred in 229 patients $(12.4 \%)$ and was unrelated to case frequency but was associated with severe foraminal stenosis $(P<.01)$. All 229 patients improved with supportive care. Facet or pedicle fractures did not occur in this series.

There were 26 acute postoperative emergency room visits by patients, 10 of which were sent home 
Table 2. Acute care emergency room visits.

\begin{tabular}{lr}
\hline Patients & No. $(\mathbf{\%})$ \\
\hline Sent home from emergency room after pain control & $10(0.54)$ \\
Dysethetic leg pain & $9(0.49)$ \\
Wound infection & $2(0.11)$ \\
Incisional pain & $5(0.27)$ \\
Admissions subtotal & $16(0.87)$ \\
Total emergency room visits & $26(1.41)$ \\
\hline
\end{tabular}

after reassurance and successful management of dysesthetic leg pain (Table 2). However, 16 patients were readmitted to a hospital over the 9-year study period $(0.87 \%)$ : 9 for dysesthetic leg pain, 2 for wound infections (1 superficial and 1 discitis), and 5 for poorly controlled incisional pain. Of the 16 admitted patients, 10 patients received a postoperative MRI scan, whereas another 3 had a postoperative CT scan as part of their postoperative work up. None of these advanced imaging studies prompted any change in management.

Additional chronic care emergency room visits occurred in another 9 patients. Four of these 9 patients were seen in the emergency room for poor pain control of underlying low back pain syndrome within 6 months from their index transforaminal decompression procedure. Two of these 4 patients were admitted to the hospital for pain control. The remaining 5 patients were seen within the 6 weeks to 6 months postoperative care interval for other conditions unrelated to the spine.

\section{DISCUSSION}

This study shows that it is feasible to perform outpatient transforaminal lumbar decompression surgery with comparable clinical outcomes in an ASC on patients who have sciatica-type low back and leg pain with lower readmission rates to either an emergency room or a hospital when compared to traditional microdiscectomy. Acute care readmission rates for outpatient ambulatory open or miniopen microdiscectomy have been reported between 4.1 and $5.8 \%{ }^{1,2,39}$ The lower readmission rate after transforaminal lumbar decompression may be at least in part explained with a more direct and anatomical approach to the compressive pathology with less exposure-related pain. Other contributing factors may be related to technological advances with endoscopic lumbar spine surgery systems allowing for more complex bony spinal stenosis problems to be treated endoscopically. Together with a general shift from inpatient to outpatient spinal surgery motivated by both patients and insurance providers, it is not surprising that endoscopic outpatient lumbar spinal surgery has been become well accepted and is done in many centers around the world. ${ }^{40}$ The clinical outcomes observed in this study with the endoscopic transforaminal decompression for extruded or contained herniated disc, as well as for spinal stenosis-related sciatica symptoms, are certainly comparable to previously published outcome studies. ${ }^{41}$ Success rates ranging from 70 to $80 \%$ have been reported as more realistic when outcomes were analyzed in terms of VAS leg $(80 \%)^{2}$ and back pain $(77 \%)^{2}$ relief, reduction in Oswestry Low Back Disability Index $(78 \%){ }^{2}$ overall satisfaction with surgery outcome $(76 \%){ }^{2}$ return to normal daily activities $(65 \%),{ }^{2}$ and work $(61 \%){ }^{2}$

Another novel aspect of the use of endoscopic transforaminal decompression surgery for foraminal and lateral recess stenosis lies in its simplicity. It is an elegant method to treat the patient's lumbar radiculopathy. Rather than taking the patient to a hospital to carry out a lumbar laminectomy microdiscectomy, the endoscopic decompression procedure is done in an outpatient setting, often under local anesthesia and sedation, with reduced cost, ${ }^{9-13}$ better patient acceptance due to fewer anesthesiarelated problems (postoperative nausea), ${ }^{42}$ and equivalent favorable clinical outcomes. ${ }^{9-13}$ Although not formally analyzed in this feasibility study, patient satisfaction with outpatient surgery has been reported as being higher than when the same surgery is done in an inpatient setting. Patient acceptance of a smaller targeted outpatient procedure carried out through smaller incisions is typically higher, presumably because of lower blood loss, fewer complications, earlier narcotic independence from surgery, and earlier return to desired activities and work. 9,40

Except for extruded disc herniations, essentially all patients were treated for lateral recess stenosis. Given excellent and good outcomes as measured with Macnab criteria in the majority of patients following the transforaminal decompression procedure, this author concludes that it can be effectively done in an ASC. In fact, similar outcomes should be expected as if the patient had the transforaminal decompression surgery as an inpatient. ${ }^{35,36}$ As sciatica and neurogenic claudication refractory to conservative care was the main reason for surgical intervention, reduction of leg pain was analyzed 
using a VAS for leg pain as one of the primary outcome measures. There was a significant improvement in the VAS and the clinical outcome at final follow up, suggesting that the outpatient transforaminal endoscopic decompression procedures is effective in the majority of patients who had unrelenting sciatica and claudication symptoms prior to surgery. Our clinical outcomes as measured by modified Macnab criteria are comparable to success rates reported by patients undergoing laminectomy for spinal stenosis. ${ }^{27,41}$

In this study, we employed a previously published radiographic classification system ${ }^{29-31}$ in the preoperative decision making in patients with symptomatic foraminal stenosis, which was shown to correlate with clinical outcomes according to the modified Macnab criteria. ${ }^{28}$ The importance of preoperative planning of transforaminal endoscopic removal of herniated discs has been stressed by Lee et al, who suggested a classification based on the location of a migrated disc fragment. ${ }^{30}$ Lee's classification systems as well as radiographic classification systems of lumbar spinal stenosis ${ }^{29}$ were successfully employed in one of the author's previously published studies, and their use was shown to correlate with favorable clinical outcomes by identifying high-risk patients that may not improve with the transforaminal decompression surgery or have a high propensity to developing problems postoperatively. ${ }^{9}$ Furthermore, our study also showed that, in skilled hands, contemporary lumbar endoscopic decompression systems can allow more sophisticated endoscopic decompression surgeries for both herniated disc and spinal stenosis.

This large patient study carried out over a 9-year period with 1839 patients enrolled showed that serious complications with the procedure are uncommon. Only 2 patients had intraoperative durotomies (incidence $0.1 \%$ ), which were managed successfully without additional surgery and, thus, were ultimately inconsequential and did not lead to any acute care admissions to an emergency room or hospital. The 2 patients with transitory foot drop were sent home from the ASC with a prescription for an AFO and ultimately did well with "handson" outpatient management, presumably due to the robust preoperative education process preparing the patient for the rare but possible complication and close clinical follow up. Admission to an emergency room or hospital from the ASC was deemed unnecessary in both patients since the directly visualized endoscopic decompression procedure was done for lateral recess stenosis without nerve root injury. Residual compression due to retained or migrated disc fragments as a result of manipulation during the decompression surgery was not suspected, and postoperative neuropraxia was the most likely explanation. Another 2 patients suffered from postoperative infections, 1 from a superficial wound infection and another patient from discitis. Both patients were admitted for work up and started on antibiotics. The patient with discitis was successfully treated with 6 weeks of intravenous antibiotics. Neither patient had any further known sequelae to date. Other clinical problems, extravasations of irrigation fluid into the subcutaneous tissues, spinal headaches, reherniation, or failure of pain relief from the index endoscopic decompression surgery also did not lead to any acute care admissions. Additional acute and chronic care admissions of patients who coped poorly with incisional pain or the underlying low back pain syndrome (14 patients) and for problems unrelated to the spine ( 5 patients) were also uncommon and rare.

By far, the most common reason for an acute care readmission after outpatient endoscopic transforaminal decompression for herniated disc or spinal stenosis is unrelenting dysesthetic leg pain due to irritation of the DRG. It typically involves the exiting nerve root, and the patient may present with new onset radicular pain in a different dermatomal distribution after an initial pain free "honeymoon" period of 3 to 14 days. It is uncommon for DRG irritation to become symptomatic after 14 days postoperatively. Other problems, such as a reherniation or infection, should be considered in the differential diagnosis if the patient presents to an emergency room or is admitted to a hospital more than 2 weeks postoperatively for unmanageable pain. However, the incidence of a postoperative nerve root irritation with $12.4 \%$ is high and occurred in 229 of the 1839 operated patients. Therefore, patients should be educated preoperatively about this common postoperative problem. Analysis showed that it was unrelated to case frequency or surgeon learning curve, but associated with severe foraminal stenosis $(P<.01)$ at a statistically relevant level. Our data show that patients exposed to the additional nerve root manipulation required during the foraminoplasty 
are prone to developing a postoperative DRG irritation. They should be educated, therefore, specifically on this otherwise benign postoperative problem. The latter statement is corroborated by the fact that all 229 patients improved with supportive care. At our outpatient spine care clinic, patients are specifically told during their preoperative consultation prior to a transforaminal decompression surgery that sudden onset of new, unfamiliar postoperative dysesthetic burning leg pain commencing after an initial short period of complete pain relief is typical of a DRG irritation and does not require a visit to the emergency room unless it is associated with motor weakness or neurological dysfunction involving the bladder or bowel. Highrisk patients are given the option of an alternative decompression procedure. With this educational program in place, a clear majority of patients with postoperative DRG irritation were successfully managed in an office setting with a combination of oral medication including nonsteroidal anti-inflammatory drugs, gabapentin, or pregabalin, transforaminal epidural steroid injection and activity modification to a light walking schedule and reduced physical activity program. Patients are advised that narcotic pain medication is not an effective treatment for dysesthetic leg pain due to postoperative DRG irritation. Poorly coping patients that present to an emergency room for postoperative pain control are likely to get noncontributory MRI or CT scans that, in our patient series, failed to change clinical management. However, these studies are often ordered by emergency room physicians or admitting hospitalists, who are typically unfamiliar with the transforaminal decompression procedure prior to contacting the operating surgeon.

To this author, it was not a surprise that unrelenting dysesthetic leg pain due to DRG irritation was the most common reason for acute care visits to an emergency room and also the most common reason for readmission to an acute care hospital postoperatively. However, the readmission rate of $0.87 \%$ over the 9 -year study period observed was low compared to readmission rates reported with traditional microdiscectomy $(4.1$ to $5.8 \%))^{2}$ In comparison, patients with persistent or recurrent symptoms did not require any acute or chronic care admissions to an emergency room or hospital presumably because of persistence or recurrence of familiar pain. Our recurrence rates for extruded disc herniations $(2.7 \%)$ and failure of pain relief in patients with bony stenosis in the central canal, lateral recess, and entry zone of the neuroforamen $(4.5 \%)$ and in patients with contained disc bulges $(6.3 \%)$ was low and commensurate with previous reports. ${ }^{2,10-13,33-41}$

Besides comparable clinical outcomes with the endoscopic outpatient lumbar transforaminal endoscopic decompression surgery when compared to traditional inpatient microdiscectomy, this surgical technique bears the upside of additional cost savings that can be realized by performing the surgery in an outpatient surgery center rather than in a hospital setting, where the costs of admitting the patient are by far higher. Hospitalization may further increase cost by greater postoperative complication rates due to higher hospital acquired wound, urinary tract, and pulmonary infection rates. Additional problems may arise in the hospital from medication errors and deviation from the postoperative pain management or rehabilitation protocol as the surgeon may not have complete control over custody of the patient throughout the hospitalization.

\section{CONCLUSIONS}

Outpatient transforaminal endoscopic decompression surgery should be considered a viable alternative to inpatient open lumbar surgery to provide patients with pain relief from sciatica-type back and leg pain. This conclusion is based not only on favorable clinical results comparable to open decompression surgery, but also on the merits of lower readmission rates. These lower readmission rates ultimately translate into cost savings that, in the context of value-based health care mandated by patients, insurance providers, governmental institutions, and review boards, will afford the spinal surgeon a modern platform to better position himself/herself competitively in the dynamically changing health care environment.

\section{REFERENCES}

1. Singhal A, Bernstein M. Outpatient lumbar microdiscectomy: a prospective study in 122 patients. Can J Neurol Sci. 2002;29(3):249-252.

2. Asch HL, Lewis PJ, Moreland DB, et al. Prospective multiple outcomes study of outpatient lumbar microdiscectomy: should 75 to $80 \%$ success rates be the norm? J Neurosurg. 2002;96(1 Suppl):34-44.

3. Zahrawi F. Microlumbar discectomy. Is it safe as an outpatient procedure? Spine (Phila Pa 1976). 1994;19(9):10701074. 
4. Bookwalter JW 3rd, Busch MD, Nicely D. Ambulatory surgery is safe and effective in radicular disc disease. Spine (Phila Pa 1976). 1994;19(5):526-530.

5. Cares HL, Steinberg RS, Robertson ET, Caldini P. Ambulatory microsurgery for ruptured lumbar discs: report of ten cases. Neurosurgery. 1988;22(3):523-526.

6. Clark AJ, Safaee MM, Khan NR, Brown MT, Foley KT. Tubular microdiscectomy: techniques, complication avoidance, and review of the literature. Neurosurg Focus. 2017;43(2):E7.

7. Overdevest GM, Peul WC, Brand R, et al; Leiden-The Hague Spine Intervention Prognostic Study Group. Tubular discectomy versus conventional microdiscectomy for the treatment of lumbar disc herniation: long-term results of a randomised controlled trial. J Neurol Neurosurg Psychiatry. 2017;88(12):1008-1016.

8. Soriano-Sánchez JA, Quillo-Olvera J, Soriano-Solis S, et al. Microscopy-assisted interspinous tubular approach for lumbar spinal stenosis. J Spine Surg. 2017;3(1):64-70.

9. Lewandrowski KU. "Outside-in" technique, clinical results, and indications with transforaminal lumbar endoscopic surgery: a retrospective study on 220 patients on applied radiographic classification of foraminal spinal stenosis. Int $J$ Spine Surg. 2014;8.

10. Yeung AT, Yeung CA. Minimally invasive techniques for the management of lumbar disc herniation. Orthop Clin North Am. 2007;38(3):363-372.

11. Tsou PM, Alan Yeung C, Yeung AT. Posterolateral transforaminal selective endoscopic discectomy and thermal annuloplasty for chronic lumbar discogenic pain: a minimal access visualized intradiscal surgical procedure. Spine $J$. 2004;4(5):564-573.

12. Tsou PM, Yeung AT. Transforaminal endoscopic decompression for radiculopathy secondary to intracanal noncontained lumbar disc herniations: outcome and technique. Spine J. 2002;2(1):41-48.

13. Yeung AT, Yeung CA. Advances in endoscopic disc and spine surgery: foraminal approach. Surg Technol Int. 2003; 11:255-263.

14. Debono B, Sabatier P, Garnault V, et al. Outpatient lumbar microdiscectomy in France: from an economic imperative to a clinical standard - an observational study of 201 cases. World Neurosurg. 2017;106:891-897.

15. Hersht M, Massicotte EM, Bernstein M. Patient satisfaction with outpatient lumbar microsurgical discectomy: a qualitative study. Can J Surg. 2007;50(6):445-449.

16. Zolot J. A worsening opioid epidemic prompts action. Am J Nurs. 2017;117(10):15.

17. Cheatle MD. Facing the challenge of pain management and opioid misuse, abuse and opioid-related fatalities. Expert Rev Clin Pharmacol. 2016;9(6):751-754.

18. Hupp JR. The surgeon's roles in stemming the prescription opioid abuse epidemic. J Oral Maxillofac Surg. 2016;74(7):1291-1293.

19. Kee JR, Smith RG, Barnes CL. Recognizing and reducing the risk of opioid misuse in orthopaedic practice. $J$ Surg Orthop Adv. Winter 2016;25(4):238-243.

20. Devin CJ, Chotai S, Parker SL, Tetreault L, Fehlings MG, McGirt MJ. A cost-utility analysis of lumbar decompression with and without fusion for degenerative spine disease in the elderly. Neurosurgery. 2015;77(Suppl 4):S116S124.

21. Adogwa O, Parker SL, Shau DN, et al. Cost per quality-adjusted life year gained of revision neural decompression and instrumented fusion for same-level recurrent lumbar stenosis: defining the value of surgical intervention. $J$ Neurosurg Spine. 2012;16(2):135-140.

22. O'Lynnger TM, Zuckerman SL, Morone PJ, Dewan MC, Vasquez-Castellanos RA, Cheng JS. Trends for spine surgery for the elderly: implications for access to healthcare in North America. Neurosurgery. 2015;77(Suppl 4):S136-S141.

23. Hignite J. Understanding the impact of the CMS 2017 ASC payment rule on spine procedures. Becker Spine Rev. 2016. https://www.beckersspine.com/spine/item/34156-understand ing-the-impact-of-the-cms-2017-asc-payment-rule-on-spine-pro cedures.html. Accessed May 15, 2018.

24. Siddiqi A, White PB, Mistry JB, et al. Effect of bundled payments and health care reform as alternative payment models in total joint arthroplasty: a clinical review. J Arthroplasty. 2017;32(8):2590-2597.

25. Sullivan R, Jarvis LD, O'Gara T, Langfitt M, Emory C. Bundled payments in total joint arthroplasty and spine surgery. Curr Rev Musculoskelet Med. 2017;10(2):218-223.

26. Mok JM, Martinez M, Smith HE, et al. Impact of a bundled payment system on resource utilization during spine surgery. Int. J Spine Surg. 2016;10:19.

27. Sengupta DK, Herkowitz HN. Lumbar spinal stenosis. Treatment strategies and indications for surgery. Orthop Clin North Am. 2003;34(2):281-295.

28. Macnab I. Negative disc exploration. An analysis of the causes of nerve-root involvement in sixty-eight patients. $J$ Bone Joint Surg Am. 1971;53(5):891-903.

29. Lee CK, Rauschning W, Glenn W. Lateral lumbar spinal canal stenosis: classification, pathologic anatomy and surgical decompression. Spine (Phila Pa 1976). 1988;13(3):313320 .

30. Lee S, Kim SK, Lee SH, et al. Percutaneous endoscopic lumbar discectomy for migrated disc herniation: classification of disc migration and surgical approaches. Eur Spine $J$. 2007;16(3):431-437.

31. Hasegawa T, An HS, Haughton VM, Nowicki BH. Lumbar foraminal stenosis: critical heights of the intervertebral discs and foramina. A cryomicrotome study in cadavera. $J$ Bone Joint Surg Am. 1995;77(1):32-38.

32. Pfirrmann CW, Metzdorf A, Zanetti M, Hodler J, Boos N. Magnetic resonance classification of lumbar intervertebral disc degeneration. Spine. (Phila Pa 1976). 2001;26(17):18731878.

33. Hoogland T, Schubert M, Miklitz B, Ramirez A. Transforaminal posterolateral endoscopic discectomy with or without the combination of a low-dose chymopapain: a prospective randomized study in 280 consecutive cases. Spine (Phila Pa 1976). 2006;31(24):E890-E897.

34. Schubert M, Hoogland T. Endoscopic transforaminal nucleotomy with foraminoplasty for lumbar disk herniation. Oper Orthop Traumatol. 2005;17(6):641-661.

35. Kambin P, Casey K, O'Brien E, Zhou L. Transforaminal arthroscopic decompression of lateral recess stenosis. J Neurosurg. 1996;84(3):462-467.

36. Kambin P, O'Brien E, Zhou L, Schaffer JL. Arthro- 
scopic microdiscectomy and selective fragmentectomy. Clin Orthop Relat Res. 1998;(347):150-167.

37. Kim MJ, Lee SH, Jung ES, et al. Targeted percutaneous transforaminal endoscopic diskectomy in 295 patients: comparison with results of microscopic diskectomy. Surg Neurol. 2007;68(6):623-631.

38. Ahn Y, Lee SH, Park WM, Lee HY, Shin SW, Kang HY. Percutaneous endoscopic lumbar discectomy for recurrent disc herniation: surgical technique, outcome, and prognostic factors of 43 consecutive cases. Spine (Phila Pa 1976). 2004;29(16):E326-E332.

39. Fallah A, Massicotte EM, Fehlings MG, et al. Admission and acute complication rate for outpatient lumbar microdiscectomy. Can J Neurol Sci. 2010;37(1):49-53.

40. Gibson JN, Cowie JG, Iprenburg M. Transforaminal endoscopic spinal surgery: the future 'gold standard' for discectomy? A review. Surgeon. 2012;10(5):290-296.

41. Fokter SK, Yerby SA. Patient-based outcomes for the operative treatment of degenerative lumbar spinal stenosis. Eur Spine J. 2006;15(11):1661-1669.

42. Shaikh S, Chung F, Imarengiaye C, Yung D, Bernstein M. Pain, nausea, vomiting and ocular complications delay discharge following ambulatory microdiscectomy. Can J Anaesth. 2003;50(5):514-518.

Disclosures and COI: The authors received no funding for this study and report no conflicts of interest. The views expressed in this article represent those of the author and no other entity or organization.

Corresponding Author: Kai-Uwe Lewandrowski, MD, 4784 E. Camp Lowell Drive, Tucson, AZ 85712. Email: business@tucsonspine.com.

Published 15 August 2018

This manuscript is generously published free of charge by ISASS, the International Society for the Advancement of Spine Surgery. Copyright (C) 2018 ISASS. To see more or order reprints or permissions, see http://ijssurgery.com. 